\title{
SFFT based ISAR Imaging
}

\author{
Yu-Zhou Gong ${ }^{\dagger}$ and Tao Hong, \\ Electronic Engineering, Beihang University, \\ Beijing, 100191, China \\ E-mail: greatgongyuzhou@126.com \\ www.buaa.edu
}

\begin{abstract}
Basing on the characteristics of radar signal and the algorithms' applicability and performance of different algorithms, this paper selects scientifically the most suitable option for radar imaging among these four sparse fast Fourier transform (SFFT) algorithms: SFFTv1,SFFTv2, SFFTv3 and PS; Evaluates each algorithm's accuracy through simulation; Applies SFFT to Inverse Synthetic Aperture Radar(ISAR) imaging program for the stepped frequency radar; Through an experiment, author studies SFFT's imaging efficiency and gets a conclusion that: SFFT is able to reduce the run time into half at a low loss of image quality.

Keywords: Sparse Fast Fourier Transform(SFFT); Radar Imaging; ISAR; Inverse Synthetic Aperture Radar(ISAR);Stepped frequency radar.
\end{abstract}

\section{Introduction}

With the development of modern military, requirement for radar imaging is rising. Decreasing run-time effectively without losing too much image quality has always been the research emphasis of this field. Recently, a serial of new types of sparse fast Fourier transforms has been developed. The run-time of SFFT for sparse signal is half less than that of the traditional FFT. For those radar imaging in which Fourier transform accounts for a heavy proportion, SFFT provides undoubtedly a new method to decrease run-time and realize real-time imaging. This paper will firstly discuss about existing SFFT algorithms from which the most suitable one will be selected out, then, evaluate the accuracy of different algorithms by simulation. Taking the inverse synthetic aperture radar implemented by pulse to pulse frequency shifting system for example, through experiment this paper studies the changes brought by inserting SFFT into ISAR imaging.

\section{Current research}

Researchers of MIT put forward a simple and practical sparse fast Fourier transform algorithm (SFFTv1) in 2012[1], promoting the development of this 
filed. Through a simple operation of "frame" dividing that divides a Fourier transform whose length is $\mathrm{n}$ into many shorter DFT, this algorithm has reduced computational complexity to $O(\log n \sqrt{n k \log (n)})$ (n stands for the length of signal while $\mathrm{k}$ is the degree of the sparsity). Researchers put forward two improved algorithms later: SFFTv2[1] and SFFTv3[3], enabling the computational complexity of SFFT decreasing to $\mathrm{O}\left(\mathrm{k}^{*} \operatorname{logn}\right)$. At the same time, transformed algorithms used in various fields are developed, such as PS (Phase Shifted) for continuous Fourier transform[4] and 2D-SFFT[5].

Having been developing for more than one century, radar imaging technology is being more and more mature. And in the field of stepped frequency, inverse synthetic aperture radar (ISAR) that mainly studied by this paper, radar parameters designing system for different situations is well improved[6]. The successful application of target extraction algorithm ensures ISAR a considerable range resolution[7]; While the application of minimal entropy theory in motion compensation also helps a lot with the improvement of image quality of ISAR imaging for fast moving objects[8]. This paper adopts the classic RD(Range Doppler) algorithm of ISAR imaging[9]

On utilizing signal sparsity to improve the efficiency of radar imaging, studies mainly base on compressed sensing theory[10] but few of them uses SFFT algorithm.

\section{Introduction of SFFT algorithm and studies of accuracy in noisy case through simulation}

\subsection{Introduction of SFFT algorithms}

SFFT (sparse fast Fourier transform) serves as a fast Fourier transform algorithm that uses specially for sparse signal. SFFT field has developed a lot in recent years and many algorithms have been put forward. This paper mainly studies four of them: SFFTv1, SFFTv2, SFFTv3 and PS (Phase Shift).

SFFTv1 is a probabilistic algorithm that calculates discrete Fourier transform[1]. Firstly, it divides the signal, with a length of N, into B sections. Secondly, basing on theorem 3.1, to calculate the FFT values at the edge of the divided sections, it makes an FFT with length B instead of N. Then, it disorders spectrum through theorem 3.2, giving every point on the spectrum a chance to be calculated. Repeating this progress for many times (depending on the sparsity $\mathrm{K}$ ), algorithm will have a chance to get all those points whose value is definitely higher than zero in the signal spectrum.

Theorem 3.1:For a signal $\mathrm{X}$ with length $\mathrm{n}$, for any positive integer $\mathrm{B}$ that can be divided exactly by $n$, define $y$ : 


$$
\forall i \in[1, \ldots, B], y_{i}=x_{i n / B}
$$

Then:

$$
\forall i \in[1, \ldots, B], y_{i}=x_{i n / B}
$$

Theorem 3.2:For a signal whose length is $\mathrm{n}$, and two random positive integers $\sigma$ and $\tau$ with:

$$
\begin{gathered}
\operatorname{gcd}(\sigma, n)=1 ; \\
\tau<=n ;
\end{gathered}
$$

Define the permutation of spectra as following:

$$
\left(P_{\sigma, \tau} x\right)_{i}=x_{(\sigma i+\tau) \bmod n}
$$

Then:

$$
\left(P_{\sigma, \tau} x\right)_{\sigma i}=x_{i} \exp (2 \pi j / n * \tau i)
$$

Nevertheless, an obvious shortcoming is found in this algorithm: the way it seeks for the point whose spectrum value is higher than zero, is very much blind and random. The improvement of the calculating efficiency is very limited. In addition, this algorithm is applicable only for certain combinations of N and $\mathrm{K}$.

Developed as the improved version of SFFTv1, SFFTv2[1] shares the same principle with SFFTv1. However, to reduce errors and computational complexity, SFFTv2 utilizes Mansour window[2] when seeking non-zero frequency spectrum point. Experiments show that : the run-time of SFFTv2 is apparently shorter than SFFTv1. However, it is still restricted by certain combinations of $\mathrm{N}$ and $\mathrm{K}$.

SFFTv3 is another improved version of these two algorithms[3]. All of these three algorithms divide signal into B sections, but differences lies in that: SFFTv1 and SFFTv2 seek for the non-zero points randomly while SFFTv3 determines directly the position of non-zero points in each sections through two permutations of spectra with the same $\sigma$ and different $\tau$ (see theorem 3.2). Another advantage of SFFTv3 is that it deletes these found spectrum values from the signal, avoiding calculating a same point repeatedly. However, the biggest defect of SFFTv3 is: when two or more points that bigger than zero lie in a same section, the estimation for non-zero spectrum value could become inaccurate, which can only be alleviated by adding the amount of divided sections B. Therefore, this algorithm is suitable only for exactly sparse signal, noiseless case. 
As a continuous Fourier transform algorithm, PS (Phase Shift) has a very similar principle with SFFTv3, but when seeking for spectra points that bigger than zero, it uses two different sampling operations instead of spectra permutations[4].

Below is comparison of the mentioned four algorithms :

Table 1 Comparison of computational complexity of four SFFT algorithms

\begin{tabular}{c|c}
\hline Algorithms & Complexity \\
SFFTv1 & $O(\log n \sqrt{n k \log (n)})$ \\
SFFTv2 & $O\left(\log n \sqrt[3]{n k^{2} \log n}\right)$ \\
SFFTv3 & $O(k \log n)$ \\
PS & $O(k \log k)$
\end{tabular}

PS shows an excellence in lessening computational complexity. However, FFT used in radar imaging are all discrete ones, PS is only helpful when conduct interpolation in discrete signal, but it is hardly a proper option because the computational complexity of interpolation is huge.

Following is the comparison of running time of SFFTv1, SFFTv2 and SFFTv3 (on the same platform):

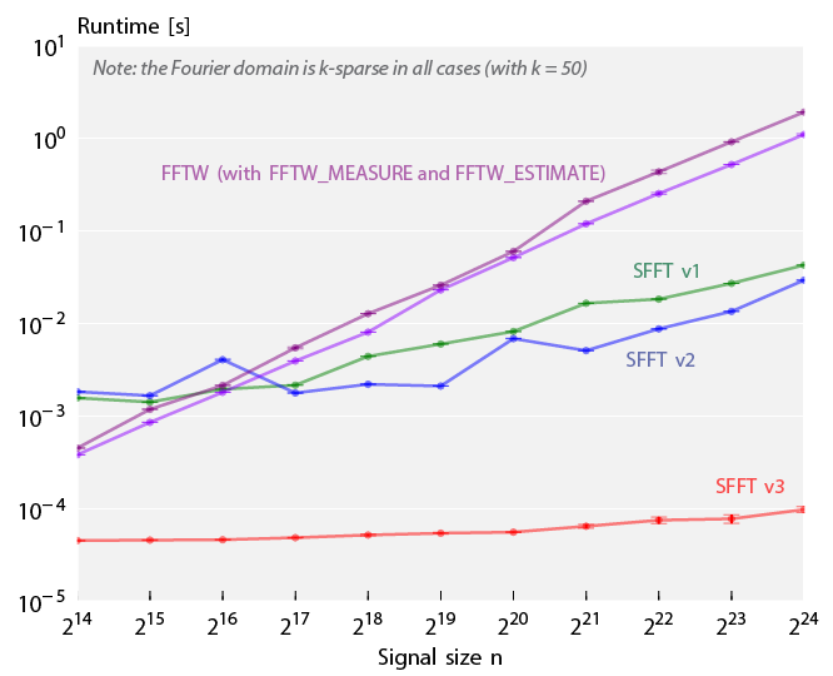

Figure 1: Comparison of the run-time of SFFTv1, SFFTv2, SFFTv3 and FFT at different signal length.

From above we can tell that: compared to the first two algorithms, SFFTv3 shows a brilliant calculating efficiency which climbs with the increasing length. Nevertheless, radar echo signal in real world can never be exactly sparse or noiseless, making the accuracy of SFFTv3 an unverified factor. Besides, 
SFFTv1 and SFFTv2 are more efficient than FFT only when signal length is bigger than 217 .

\section{Studies of the accuracy of SFFT in noisy case through simulation}

1. For an exactly sparse signal with length $N=16384$ and sparsity $K=10$, the non-zero spectrum points distribute uniformly and randomly, for example:

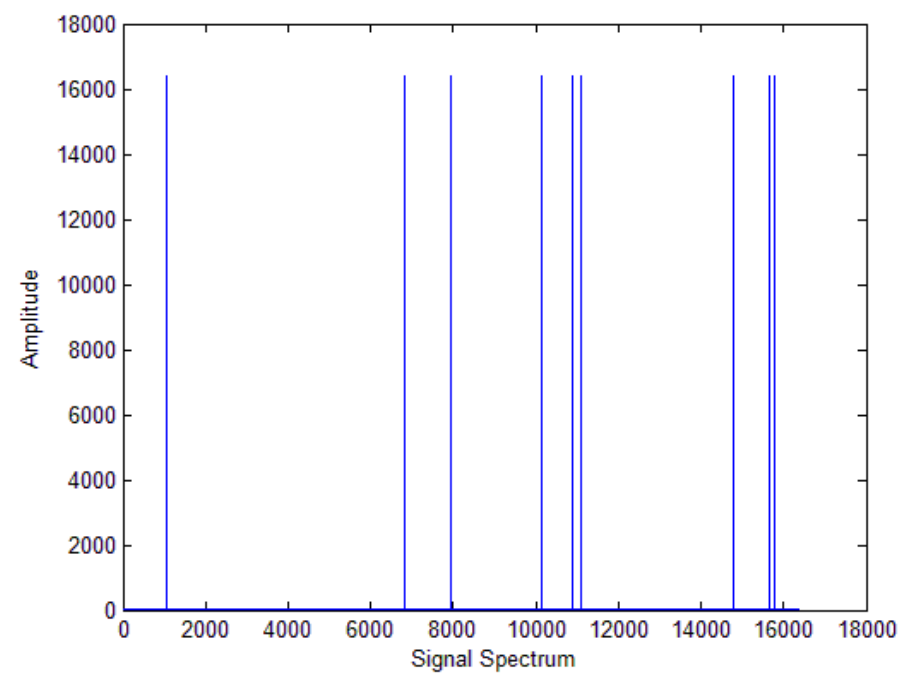

Figure 2. Example spectrum of simulation signal

2.Get time-domain signal through IFFT

3.Add noise to time-domain signal

4.Utilize SFFTv1, SFFTv2 and SFFTv3 to calculate FFT of signal with noise, recording the amount of points accurately calculated (5\% tolerance)

5.Repeat 1-4 100 times

We have obtained the possibility of finding and calculating accurately these spectrum points at different signal noise ratio $(\mathrm{SNR}=0,5,10,15)$ :

Table 2. Comparison of the accuracy ofSFFTv1, SFFTv2 and SFFTv3

\begin{tabular}{cccc}
\hline & SFFTv1 & SFFTv2 & SFFTv3 \\
\hline SNR=0 & $75 \%$ & $78 \%$ & $0 \%$ \\
SNR=5 & $95 \%$ & $93 \%$ & $0 \%$ \\
SNR $=10$ & $100 \%$ & $100 \%$ & $0 \%$ \\
SNR $=15$ & $100 \%$ & $100 \%$ & $0 \%$ \\
\hline
\end{tabular}


It can be seen that: SFFTv3, offering a low accuracy when calculating signal with noise, is not a suitable algorithm for radar signal who has a smaller noise-signal ratio in real world. Therefore, radar imaging algorithms adopted in this paper are mainly SFFTv1 and SFFTv2.

SFFTv1 and SFFTv2 has similar probability in finding non-zero spectrum points. SFFTv2, however, will actually introduce additional noise in signal, which will produce noise point in the image.

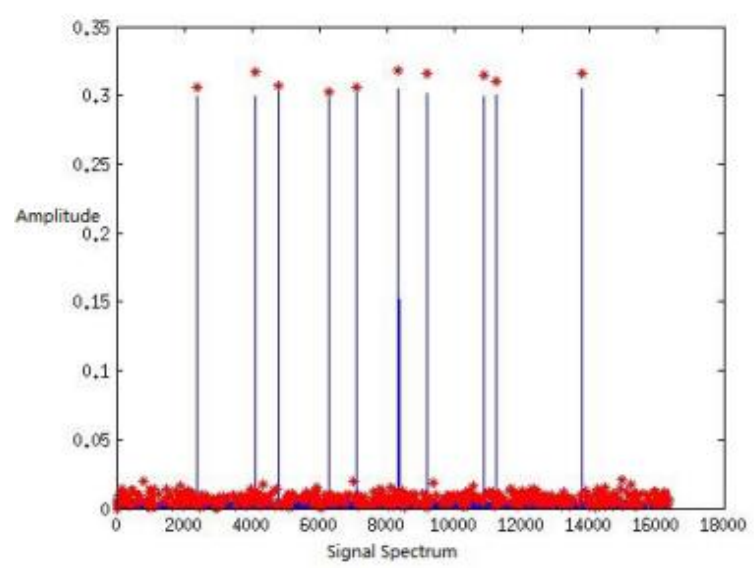

Figure 3 Example of additional noise of SFFTv2

\section{Experiment}

The experiment uses pulse to pulse frequency shifting outdoor RCS measurement system[11]. The object of imaging is a sphere with radius $0.1 \mathrm{~m}$.

The signal sent by radar is stepped frequency signal, with initial frequency $\mathrm{fO}=13.5 \mathrm{GHz}$, final frequency $=15.5 \mathrm{GHz}$ and the frequency step deltaf $=10 \mathrm{kHz}$. The object is $1.0142 \mathrm{~m}$ away from radar.

We have obtained one-dimension images through traditional FFT and SFFTv1 algorithm, as shown in Figure 4.

The distance obtained by SFFT is $1.0139 \mathrm{~m}$. The final length of signal is 262144 , longer than 217, which is the condition that SFFT's running time is shorter than that of FFT.

Calculating repeatedly for 1000 times, we record the running time of FFT and SFFTv1, as shown in Table 3. 


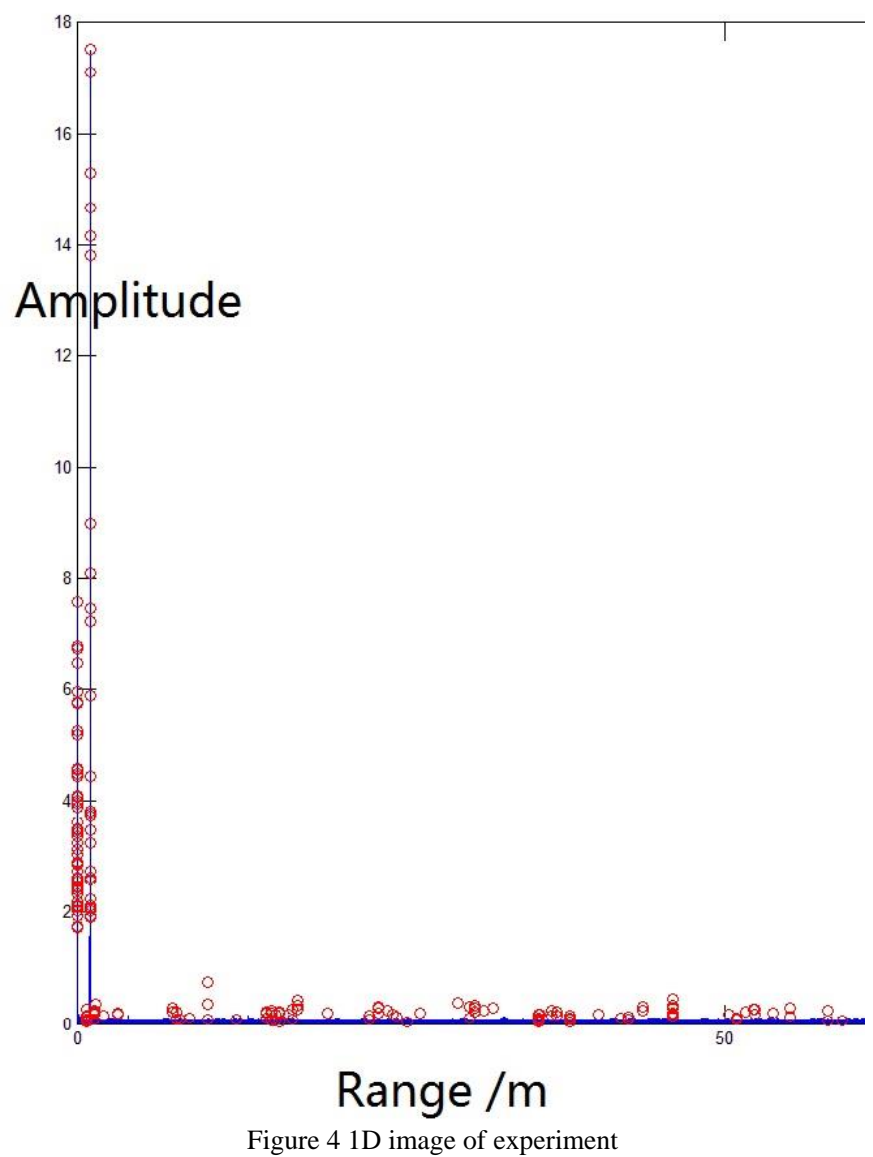

Table 3 Comparison of running time of FFT and SFFTv1 for 1000 one-dimension images

\begin{tabular}{cc}
\hline Algorithm & Running time \\
\hline FFT & $12.925 \mathrm{~s}$ \\
SFFT & $6.205 \mathrm{~s}$ \\
\hline
\end{tabular}

\section{Conclusion}

The result shows that: SFFT algorithm can reduce effectively the calculating time of ISAR imaging - its calculating time only accounts for half of the run time of the traditional Fourier transform algorithm, and the longer the signal is (>217), the bigger the advantage of SFFT will be, for a stepped frequency system with bigger bandwidth and smaller frequency step, SFFT is a proper 
choice to help to decrease calculating time. As for the accuracy, SFFT would bring some errors, but when it comes to the estimation of non-zero points, SFFT is able to calculate precisely the distance of objects $(0.03 \%$ error $)$.

\section{References}

1. H.Hassanieh, P. Indyk, D. Katabi, and E. Price. Simple and practical algorithm for sparse Fourier transform. SODA, 2012.

2. Y.Mansour. Randomized interpolation and approximation of sparse polynomials. ICALP, 1992.

3. Haitham Hassanieh, Piotr Indyk, Dina Katabi, and Eric Price. Nearly optimal sparse Fourier transform. In STOC, pages 563 - 578, 2012.

4. David Lawlor, Yang Wang, and Andrew Christlieb. Adaptive Sub-Linear Time Fourier Algorithms. 2012

5. Badih Ghazi, Haitham Hassanieh, Piotr Indyk, Dina Katabi, Eric Price, Lixin Shi Allerton. Sample-Optimal Average-Case Sparse Fourier Transform in Two Dimensions, October 2013.

6. Ren Li-xiang, Mao Er-ke. Study on HPRF Pulsed Doppler Stepped Frequency Radar System. 2006 CIE International Conference on Radar.

7. ChaoJie Liang, Yang Li, DingHong Lu, Teng Long. An improved target extraction algorithm for stepped frequency chirp signal. Radar Conference 2013, IET International.

8. Daiyin Zhu, Ling Wang, Yusheng Yu. Robust ISAR Range Alignment via Minimizing the Entropy of the Average Range Profile. IEEE Geoscience and Remote Sensing Letters, 2009.

9. LONG Teng, HAN Yue-qiu, MAO Er-ke. Digital Signal Processing of Stepped Frequency Radar. Acta Aeronautica et Astronautica Sinica, Vol22, June 2001.

10. Lee C.Potter, Emre Ertin, Jason T.Parker, Müjdat Cetin. Sparsity and Compressed Sensing in Radar Imaging. Proceedings of the IEEE, Volume:98, Issue: 6, Feb 2010.

11. PENG Gang, LIANG Yi, TIAN Jinjun, XUE Minghua. Simplified Of High Range Resolution Pulse to Pulse Frequency Shifting Outdoor RCS Measurement Device. Modern Radar Vol27 No7, July 2005. 